mice were assessed for right ventricular systolic pressure (RVSP) and right ventricular hypertrophy.

Results Reduced caveolae and depth of invaginations were observed in idiopathic PAH patients and individuals with a BMPR-II mutation when compared to controls. Interestingly, an individual with a BMPR-II mutation without disease had similar levels to controls. Furthermore, cavin-2 protein expression was decreased in cells from individuals with pulmonary hypertension, but other caveolae components appeared unaffected. In the absence of a disease stimulus caveolin-1 and cavin-2 knockout mice did not develop pulmonary hypertension although slightly elevated RVSP was observed.

Conclusions Our preliminary data suggests that caveolae formation is dysregulated in cells from individuals with pulmonary hypertension. In addition, reduced levels of cavin- 2 could play a significant role in the decreased number of caveolae. Cavin-2 and caveolae generation could therefore be novel therapeutic targets for pulmonary hypertension.

\section{S139 THE ROLE OF ENDOTHELIN RECEPTORS (ETRA/B) IN FIBROCYTE DIFFERENTIATION}

${ }^{1}$ SLI Trinder, 'X Shi-wen, 'B Ahmed Abdi, ' $C P$ Denton, ${ }^{2}$ DC Budd, 'DJ Abraham, 'AM Holmes; 'Centre for Rheumatology \& Connective Tissue Diseases, UCL Medical School, Royal Free Campus, London, UK; ${ }^{2}$ Respiratory Drug Discovery, Inflammation, Hoffmann-La Roche Inc., Nutley, USA

\subsection{6/thoraxinl-2013-204457.146}

Introduction Scleroderma (SSc) is an autoimmune connective tissue disease of unknown aetiology. Pulmonary involvement including the development of pulmonary arterial hypertension (PAH) is characterised by vascular remodelling, collagen deposition and expression of connective tissue growth factor (CTGF). $\mathrm{CD}_{14}{ }^{+}$monocytes can differentiate into spindle shaped cells termed 'fibrocytes'. Fibrocytes express haematopoietic and mesenchymal markers including collagen, and amplify inflammatory/ immune responses via antigen presentation and chemokine secretion. Fibrocyte differentiation is enhanced by fibrogenic cytokines including PDGF. The role fibrocytes play in promoting $\mathrm{PAH}$ in SSc is unknown.

Methods CD14 ${ }^{+}$PBMCs were isolated from SSc and healthy donor blood. Fibrocyte differentiation in the presence of MCSF and/or ET-1 was assessed after 14 days. The effect of endothelin receptor (ETR) antagonists (selective/dual) on fibrocyte differentiation $(\mathrm{n}=6)$ was investigated. SSc and control fibrocyte secretomes were assessed by ELISA $(n=6)$, and the effects on fibroblast-mediated gel contraction determined.

Results MCSF and ET-1 alone and in combination induced fibrocyte differentiation $(\mathrm{P}<0.05)$. SSc fibrocytes exhibited enhanced differentiation from CD14 ${ }^{+}$PBMCs than healthy control donors in response to MCSF $(\mathrm{P}<0.05)$, ET-1 $(\mathrm{P}<0.05)$ and in combination $(\mathrm{P}<0.01)$. ETR antagonists $\mathrm{BQ} 123\left(\mathrm{ETR}_{\mathrm{A}}\right)$, $\mathrm{BQ788}\left(\mathrm{ETR}_{\mathrm{B}}\right)$ and Bosentan $\left(\mathrm{ETR}_{\mathrm{A} / \mathrm{B}}\right)$ inhibited MCSF induced fibrocyte differentiation. CTGF secretion was elevated in SSc compared to control fibrocytes $(\mathrm{P}<0.05)$ cultured with MCSF. Conditioned media from SSc fibrocytes promoted gel contraction by control pulmonary fibroblasts $(\mathrm{P}<0.05)$.

Discussion CD14 ${ }^{+}$SSc PBMCs readily differentiate into fibrocytes in response to ET-1 and MCSF via $\mathrm{ETR}_{\mathrm{A}}$ and $\mathrm{ETR}_{\mathrm{B}}$. Our data suggests fibrocytes contribute to the development of $\mathrm{PAH}$ in SSc via a paracrine mechanism modulating the functional activities of resident tissue fibroblasts.
S140 BMPR-II DEFICIENCY LEADS TO AN INCREASE IN EGG DEPOSITION AND CYTOKINE RELEASE IN THE LUNGS OF MICE CHRONICALLY INFECTED WITH SCHISTOSOMIASIS

A Crosby, E Soon, F Jones, M Southwood, L Haghighat, M Toshner, T Raine, I Horan, P Yang, A Davenport, DW Dunne, NW Morrell; Cambridge University, Cambridge, UK

\subsection{6/thoraxjnl-2013-204457.147}

Rationale and Objectives Schistosomiasis is the world-wide leading cause of pulmonary arterial hypertension (PAH) and is particularly prevalent in the third-world. More than $80 \%$ of patients with PAH in the western world have a mutation in bone morphogenetic protein type-II receptor (BMPR-II), which is a member of the transforming growth receptor-beta (TGF-b) superfamily and is important in cell proliferation and differentiation. The aim of the study was to determine if mice with a heterozygous null mutation in BMPR-II are more susceptible to schistosomiasis-induced PAH, compared to wild-type littermates. Methods Wild-type and mutant C56/BL6 mice were infected percutaneously with a low dose of S.mansoni. Non-infected WT and MUT mice were also studied. At 17 weeks post-infection right ventricular systolic pressure (RVSP) and right ventricular (RV) hypertrophy, liver and lung egg counts and body weight were measured. Pulmonary vascular remodelling and liver histology were assessed by morphometry, following immunohistochemistry. Micro-CT was performed to determine egg deposition. A macrophage phagocytosis assay was also performed.

Measurements and Main results At 17 weeks post-infection there was no significant difference in RVSP, the degree of RV hypertrophy, mean area of liver vasculature, mean number of liver vessels or liver weight between infected BMPR-II $+1+$ and BMPR-II +/- mice. However, there was a significant reduction in body weight, a significant increase in lung egg deposition and lung cytokine expression in the BMPR-II +/- mice compared to the wild-type mice 17 weeks post-infection. There was no significant difference in serum or liver cytokine levels. We saw a significant increase in pulmonary vessel wall thickness in both BMPR-II + / + and BMPR-II +/- mice infected mice, compared to their respective non-infected controls. There was no difference in the ability of macrophages from BMPR-II $+/+$ and BMPR-II +/- mice to phagocytose fluorescently tagged beads. Conclusions This study has shown that BMPR-II mutations do not predispose to schistosomiasis-induced PAH, but that there is an increased ability of the eggs to gain access into the lungs and a subsequent heightened inflammatory response. This appears not to be due to an innate difference in the liver vasculature or a defect in egg clearance by macrophages.

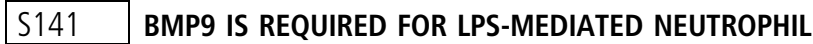 RECRUITMENT TO PAH-PATIENT DERIVED BLOOD OUTGROWTH ENDOTHELIAL CELLS WITH BMPR-II MUTATIONS}

${ }^{1} \mathrm{SL}$ Appleby, ${ }^{1} \mathrm{~J}$ Deighton, ${ }^{2} \mathrm{CM}$ Yates, ${ }^{1} \mathrm{MR}$ Toshner, ${ }^{2} \mathrm{~GB}$ Nash, ${ }^{1} \mathrm{ER}$ Chilvers, ${ }^{1} \mathrm{NW}$ Morrell; ${ }^{1}$ University of Cambridge, Cambridge, united Kingdom; ' University of Birmingham, Birmingham, United Kingdom

10.1136/thoraxjn-2013-204457.148

Introduction Heterozygous mutations in the gene encoding bone morphogenetic protein (BMP) receptor II (BMPR-II) are present in $>70 \%$ of patients with heritable pulmonary arterial hypertension (hPAH) and $15-26 \%$ of idiopathic $\mathrm{PAH}$ (iPAH) 
cases. The genetic data strongly implicates the endothelium as the initiating cell type in $\mathrm{PAH}$, and BMP9 is the major circulating ligand for BMPR-II on endothelial cells (EC). Since inflammation is emerging as a potential trigger for disease in BMPR-II mutation carriers, we hypothesised that BMP9 and BMPR-II mutation might impact on endothelial:leukocyte interaction.

Methods We employed blood outgrowth endothelial cells (BOEC) isolated from healthy donors (control-BOEC) and BOEC derived from PAH patients with BMPR-II mutations (PAH-BOEC) in this study.BOEC can be generated from venous blood and are recognised as a non-invasive source of patientderived EC. Endothelial:neutrophil interaction was studied using an in vitro flow-based model. Neutrophil recruitment to LPS (100 ng/ml; 4h)-stimulated BOECs was quantified in the presence or absence of BMP9 $(5 \mathrm{ng} / \mathrm{ml} ; 16 \mathrm{~h})$ under physiologically relevant sheer stress $(0.1 \mathrm{~Pa})$. Surface expression of E-selectin was quantified using flow cytometry, ELISA was used to assess the secretion of IL-8 and mRNA levels were quantified by qPCR.

Results LPS stimulation induced robust neutrophil recruitment to control-BOEC, whereas BMP9 had no effect.Co-stimulation with LPS and BMP9 resulted in a synergistic increase in neutrophil recruitment and control-BOEC E-selectin and IL-8 expression, compared to stimulation with LPS alone. Moreover, BMP9 stimulated the upregulation of TLR4 on control-BOEC (the receptor for LPS), thus providing a potential mechanism for the enhanced inflammatory response seen with LPS and BMP9 costimulation. In contrast to the findings in control-BOEC, LPS stimulation failed to induce neutrophil recruitment in $\mathrm{PAH}-$ BOEC. However, co-stimulation with LPS and BMP9 restored neutrophil recruitment in PAH-BOEC.

Conclusions BMP9 markedly enhances neutrophil recruitment to the endothelium in response to LPS. Deficiency in BMP9 signalling (as a consequence of BMPR-II mutations in PAH-BOEC) impairs this process, and BMP9 restores normal endothelial:neutrophil interactions in PAH-BOECs. These findings identify a hitherto unknown role for BMP9 in endothelial:neutrophil interaction and implicate this process in the pathobiology of PAH.

\section{S142 THE ROLE OF H3K27 METHYLATION IN VASCULAR ENDOTHELIAL CELL PROLIFERATION AND FUNCTION: IMPLICATIONS FOR PULMONARY ARTERIAL HYPERTENSION}

'D Shao, ${ }^{1} \mathrm{~N}$ Gambaryan, ${ }^{1} \mathrm{C}$ Meng, ${ }^{2} \mathrm{~F}$ Perros, ${ }^{2} \mathrm{M}$ Humbert, I Adcock, ${ }^{1} \mathrm{~S}$ Wort; ${ }^{1}$ Imperial College London, London, UK; ${ }^{2}$ Univ. Paris-Sud, Paris, France

\subsection{6/thoraxjnl-2013-204457.149}

Introduction and Objectives There is increasing interest in the role of epigenetic gene regulation in the pathogenesis of pulmonary arterial hypertension (PAH), a condition associated with pulmonary vascular cell proliferation. Methylation on histone H3K27 (H3K27me3) has been found to be a key regulator of development and cell homeostasis. Methylation at H3K27 can be reversed by Jumonji $\mathrm{C}(\mathrm{JmjC})$ domain-containing proteins, JMJD3. We were interested to determine the immunohistochemical distribution of $\mathrm{H} 3 \mathrm{~K} 27 \mathrm{me} 3$ and JMJD3 proteins in patients with IPAH and the effect of GSK-J4, an inhibitor of JMJD3, on markers of pulmonary vascular cell proliferation.

Methods Immunohistochemistry for JMJD3 and H3K27me3 was performed on human lung from normal healthy controls and patients with idiopathic PAH. The role of JMJD3 on cell cycle regulator (cyclin) expression in response to serum stimulation, with and without GSK-J4, was performed on normal human pulmonary artery endothelial cells (HPAECs) and RT-qPCR was then performed using QuantiTec primer assays. Chromatin Immunoprecipitation (CHiP) was performed antibody against H3K27me3 on normal HPAECs and analysed by quantitative PCR.

Results We found that the expression of JMJD3 protein is increased in the walls of pulmonary arteries of patients with idiopathic PAH compared to health control. This correlated to a decreased expression of H3K27me3. Treatment of HPAECs with GSK-J4, the inhibitor of JMJD3, in vitro, significantly increased cell cycle regulator p19, p27, CDK4 and CDK6 mRNA expression (Fig. $1, \mathrm{n}=3$ ), whilst having no effect on $\mathrm{p} 14, \mathrm{p} 16, \mathrm{p} 21$ and CDK2 (data not shown). Furthermore, CHiP using a specific H3K27me3 antibody, followed by RT-qPCR analysis, demonstrated that GSK-J4 treatment increased H3K27me3 occupation on the transcription start sites of p27 and CDK4, whilst the negative control compound GSK-J5 had no effect $(n=2)$.

Conclusion Our data suggest that JMJD3 and H3K27 histone methylation could play an important role in cell cycle progression and cell cycle exit of pulmonary vascular cells in patients with idiopathic PAH. As such drugs such as GSK-J4 may provide a therapeutic option in the future to reverse pulmonary vascular remodelling.

\section{S143 EVIDENCE THAT TYPE I INTERFERON DRIVES PULMONARY ARTERIAL HYPERTENSION}

${ }^{1} \mathrm{PM}$ George, ${ }^{1} \mathrm{E}$ Oliver, ${ }^{2} \mathrm{BE}$ Schreiber, ${ }^{2} \mathrm{AM}$ Holmes, ${ }^{3} \mathrm{M}$ Southwood, ${ }^{1} \mathrm{~S} J$ Wort, ${ }^{1} \mathrm{~N}$ Bartlett, ${ }^{4} \mathrm{P}$ Dorfmuller, ${ }^{3} \mathrm{NW}$ Morrell, ${ }^{2} \mathrm{G}$ Coghlan, ${ }^{4} \mathrm{M}$ Humbert, ${ }^{1} \mathrm{~L}$ Zhao, ${ }^{1} \mathrm{JA}$ Mitchell; ${ }^{1}$ Imperial College, London, UK; ${ }^{2}$ Royal Free Hospital, London, UK; ${ }^{3}$ Papworth Hospital NHS Trust, Cambridge, UK; ${ }^{4}$ Univ. Paris-Sud, Kremlin-Bic $\downarrow$ tre, France

\subsection{6/thoraxjnl-2013-204457.150}

Rationale There is increasing evidence of a link between interferon (IFN) and pulmonary arterial hypertension (PAH). Conditions such as HIV and Systemic sclerosis (SSc) where endogenous IFN levels are chronically elevated are strongly associated with $\mathrm{PAH}$. Furthermore, therapeutic use of type I IFN is now known to cause PAH and increase the systemic release of endothelin (ET)-1 in some patients. The link between IFN therapy and PAH was formally recognised at the 5-yearly World Symposium on Pulmonary Hypertension (Nice, Feb 2013). Our group has
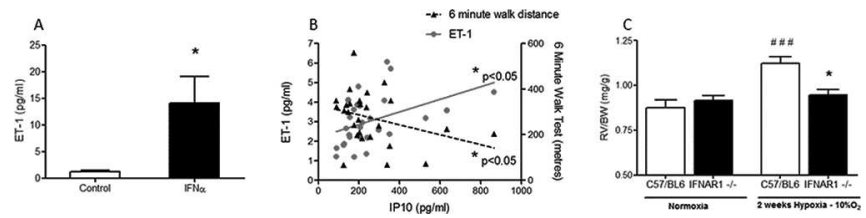

Abstract S143 Figure 1. A ET-1 release from human pulmonary artery smooth muscle cells. Data expressed as mean \pm SEM from $\mathrm{n}=\mathbf{5}$ experiments. Statistical significance determined by one-way ANOVA * $\mathbf{p}<0.05$. B Pearson Correlation between serum levels of IP10 and ET-1 $(* p<0.05)$ and IP10 and 6 minute walk test (* $\mathbf{p}<0.05)$ in 28 patients with Systemic Sclerosis associated PAH. C Mice lacking a functional type I IFN receptor (IFNAR1 $\%^{-}$) exposed to hypoxia $(10 \% \mathrm{O} 2)$ or normoxia compared to wild type (C57BI/ 6J) mice. Data presented as mean \pm SEM for $n=4-15$ mice. Right ventricular hypertrophy assessed as ratio of right ventricular (RV) mass to body weight (BW) (RV/BW). Statistical significance determined by one-way ANOVA followed by Bonferroni's multiple comparison post-test $(\# \# \# p<0.0001$ for normoxic vs. hypoxic conditions) and (*p $<0.05$ for IFNAR ${ }^{-}$- vs. $\mathrm{C} 57 \mathrm{BI} / 6 \mathrm{~J}$ mice). 